\title{
Eficiência fotossintética da cana-de-açúcar submetida à aplicação de atrazine e tebuthiuron em pré-emergência ${ }^{1}$
}

\section{Photosynthetic efficiency of sugarcane crop submitted to atrazine and tebuthiuron application in pre-emergency}

\author{
Marcelo Girotto $^{2}$, Rosilaine Araldi ${ }^{3}$, Edivaldo Domingues Velini ${ }^{4}$, Giovanna Larissa G. Cotrick \\ Gomes $^{3}$, Caio Antônio Carbonari ${ }^{4}$, Samir Paulo Jasper ${ }^{5}$, Lucia Maria Bueno Trindade ${ }^{6}$
}

Resumo - O objetivo do trabalho foi avaliar a eficiência fotossintética da cana-de-açúcar após aplicação, em pré-emergência, de dois herbicidas inibidores da fotossíntese. Para o plantio dos toletes da cana-de-açúcar (variedade SP80-3280), utilizaram-se vasos com capacidade de $8 \mathrm{dm}^{3}$. A aplicação do atrazine e tebuthiuron foi realizada através de um pulverizador estacionário instalado em laboratório e após a aplicação dos tratamentos, as plantas foram transportadas para casa-de-vegetação onde ficaram até o término do ensaio. Realizou-se a leitura da taxa de transporte de elétrons (ETR) na porção mediana das folhas mais novas de cana-de-açúcar com fluorômetro portátil sendo que os intervalos de avaliação do ETR foram de: 16, 17, 19, 25 e 30 dias após a aplicação dos herbicidas. Realizou-se ainda análise visual de fitointoxicação aos 30 dias após aplicação. Os resultados demonstraram que o tebuthiuron causou maior redução da taxa de transporte de elétrons em comparação com a atrazine. Em relação aos sintomas de fitointoxicação, não foi observada diferença entre os herbicidas testados. Ao fim do trabalho, verificou-se que a metodologia empregada com o uso do fluorômetro para medir a taxa de transporte de elétrons após a aplicação dos herbicidas foi adequada, permitindo verificar a intoxicação da cultura de cana-de-açúcar, mesmo antes de qualquer detecção visual da atuação dos herbicidas nas plantas.

Palavras-chave: Fluorômetro; Seletividade; ETR.

\begin{abstract}
The objective of this work was to assess the photosynthetic efficiency of sugarcane crop, in pre-emergency application, of two inhibiting photosynthesis herbicides. For sugar cane cuttings planting (SP80-3280 genotype), pots with a capacity of $8 \mathrm{dm}^{3}$ were used. Atrazine and tebuthiuron application was performed by using a stationary spray installed in laboratory conditions and after treatment application, plants were transported to a green house where were kept until the end of the trial. The reading of electrons transport rate (ETR) was conducted in the middle portion of the youngest leaves of sugarcane with a portable fluorometer, with intervals for ETR assessment constituted by: 16, 17, 19, 25 and 30 days after herbicide application. It was also

\footnotetext{
${ }^{1}$ Recebido para publicação em 02/03/2011 e na forma revisada em 15/08/2011.

${ }^{2}$ Eng $^{\circ}$ Agr ${ }^{\circ}$, Mestrando do Programa de Pós-graduação em Agricultura, FCA/UNESP - Botucatu/SP. E-mail: girotto@fca.unesp.br.

${ }_{3}^{3}$ Doutoranda do Programa de Pós-graduação em Agricultura, FCA/UNESP - Botucatu/SP.

${ }^{4}$ Prof. Adjunto, Depto. Produção Vegetal, FCA/UNESP - Botucatu/SP.

${ }^{5}$ Eng $^{\circ}$ Agr $^{\circ}$, Dr pelo Programa de Pós-graduação Produção Vegetal, FCA/UNESP - Botucatu/SP.

${ }^{6}$ Eng $^{\mathrm{a}}$ Agr $^{\mathrm{a}}$, Bioativa Pesquisa e Compostos Bioativos Ltda - Botucatu/SP.
} 
developed visual analysis of phytotoxiciy performed 30 days after application. The results showed that tebuthiuron herbicide caused greater reduction in electrons transport rate than atrazine. In relation to herbicide symptoms, there was no significant difference between the studied herbicides. At the end of the study it could be verified that the chosen methodology by using fluorometer to measure the electrons transport rate after herbicide application was adequate, allowing verifying sugar cane crop intoxication, even before any visual detection of herbicides performance in plants.

Key-words: Fluorometer; Selectivity; ETR.

\section{Introdução}

A cana-de-açúcar pode ter sua produtividade comprometida pela presença de plantas daninhas. Essa redução ocorre, principalmente, pela competição por nutrientes, hospedagem de pragas e doenças, além da liberação de aleloquímicos. Além disso, a presença de plantas daninhas dificulta o corte dos colmos de cana-de-açúcar na ocasião da colheita, fazendo com que o rendimento industrial decresça resultando na perda da qualidade da cana-de-açúcar (Negrisoli et al., 2004).

Segundo Freitas et al. (2004), na condição atual de produção da cana-de-açúcar, o método químico é o mais utilizado no controle das plantas daninhas em razão, principalmente, das extensas áreas cultivadas, escassez de mão-de-obra, facilidade de aplicação, custo e eficácia do tratamento, além de ser um método econômico e de alto rendimento, em comparação com os outros.

A adequada aplicação de herbicidas é fundamental para que o mesmo atinja seu alvo de modo a realizar o controle sem ocasionar problemas de fitointoxicação na cultura ou contaminação ambiental. Entretanto, devido às similaridades anatômicas e fisiológicas entre as plantas daninhas e as espécies cultivadas, os riscos de intoxicação das culturas sempre ocorrem quando se adota o controle por meio de herbicidas.

A capacidade de um determinado herbicida em eliminar as plantas daninhas que se encontram em uma cultura, sem reduzir-lhe a produtividade e a qualidade do produto final obtido, recebe o nome de seletividade. A seletividade não pode ser determinada apenas pela simples verificação de sintomas visuais de intoxicação, pois são conhecidos exemplos de herbicidas que podem reduzir a produtividade das culturas sem, no entanto, produzir-lhes efeitos visualmente detectáveis e também exemplos de herbicidas que provocam injúrias bastante acentuadas, mas que permitem a elas manifestarem plenamente seus potenciais produtivos (Negrisoli et al., 2004).

Para controlar as principais plantas daninhas e evitar os possíveis prejuízos à cultura da cana-de-açúcar, muitos herbicidas com diferentes ingredientes ativos $\mathrm{e}$ formulações estão registrados para o uso no Brasil. Dentre eles, destacam-se a atrazine e tebuthiuron (Rodrigues \& Almeida, 2005).

Os herbicidas tebuthiuron e atrazine por possuírem como mecanismo de ação a inibição do fotossitema II, se ligam a proteína D1, que é o sítio de ligação da $Q_{B}$, o qual se localiza nas membranas dos tilacóides dos cloroplastos, causando, por consequência, o bloqueio do transporte de elétrons de $Q_{A}$ para $Q_{B}$. Esta reação reduz a fixação de $\mathrm{CO}_{2}$, a produção de ATP e $\mathrm{NADPH}_{2}$, os quais são elementos essenciais para o crescimento das plantas (Breitenbach et al., 2001).

Em relação à fase de transporte de elétrons durante a fotossíntese nas plantas, tem- 
se que a luz é absorvida por pigmentos do complexo antena, que ao excitarem os elétrons, transferem energia para os centros de reação dos fotossistemas II e I (Young \& Frank, 1996). Quando ocorre excesso de energia, esta pode ser dissipada na forma de fluorescência (Krause \& Winter, 1996). Desta forma, uma das formas de monitoramento da inibição ou redução na transferência de elétrons entre os fotossistemas de plantas sob aplicação de herbicidas, que pode ser observada ainda em folhas intactas, é a fluorescência da clorofila (Maxwell \& Johnson, 2000); sendo que a redução na dissipação da energia pelo processo fotoquímico é refletida por incremento correspondente na fluorescência.

A análise da fluorescência vem sendo largamente utilizada no entendimento dos mecanismos fotossintéticos, auxiliando, principalmente, na mensuração desses mecanismos após aplicação de herbicidas (Ireland et al., 1986). A ferramenta disponível para tal mensuração é o fluorômetro, capaz de identificar rapidamente as injúrias causadas ao aparelho fotossintético, mesmo não sendo detectados visualmente os sintomas de intoxicação.
O presente trabalho teve por objetivo avaliar a eficiência fotossintética na cultura da cana-de-açúcar com aplicação de herbicidas inibidores do fotossistema II em préemergência.

\section{Material e métodos}

Foi conduzido um experimento em casa de vegetação no Núcleo de Pesquisas Avançadas em Matologia (NUPAM), pertencente à Faculdade de Ciências Agronômicas de Botucatu/SP, nas seguintes coordenadas geográficas: latitude de $22^{\circ} 07^{\prime} 56^{\prime}$ ' S, longitude de $74^{\circ} 66^{\prime} 84^{\prime}$ ' W Gr. e altitude de $762 \mathrm{~m}$.

O solo utilizado como substrato foi inicialmente seco à sombra por um período de 48 horas, e posteriormente peneirado em peneira com malha de 200 mesh, sendo submetido às análises química e física (Tabela 1). A adubação foi realizada com base nos resultados da análise do solo, em seguida, procedeu-se o plantio dos toletes de cana-deaçúcar em vasos de $8 \mathrm{dm}^{3}$ de capacidade (variedade utilizada SP80 -3280).

Tabela 1 - Análise química e física do solo utilizado no experimento com cana-de-açúcar submetida à aplicação de herbicidas em pré-emergência. Botucatu, SP, 2011.

\begin{tabular}{|c|c|c|c|c|c|c|c|c|c|}
\hline $\begin{array}{c}\mathrm{pH} \\
\left(\mathrm{CaCl}_{2}\right)\end{array}$ & $\begin{array}{c}\text { M.O } \\
\left(\mathrm{g} \mathrm{dm}^{-3}\right)\end{array}$ & $\begin{array}{c}\text { P res. } \\
\left(\mathrm{mg} \mathrm{dm}^{-3}\right)\end{array}$ & $\mathrm{K}^{+}$ & $\mathrm{Ca}^{+2}$ & $\begin{array}{r}\mathrm{Mg}^{+2} \\
(\mathrm{~m}\end{array}$ & $\begin{array}{l}\mathrm{H}^{+}+\mathrm{Al}^{+3} \\
\left.\mathrm{Ol}_{\mathrm{c}} \mathrm{dm}^{-3}\right)\end{array}$ & SB & $\mathrm{T}$ & $\mathrm{V}(\%)$ \\
\hline 4,3 & 19,0 & 1,0 & 0,6 & 10,0 & 4,0 & 5,8 & 14,6 & 73 & 21 \\
\hline \multirow{3}{*}{$\begin{array}{c}\text { Granulometria } \\
(\%)\end{array}$} & \multicolumn{9}{|c|}{ Classe de solo } \\
\hline & \multirow[b]{2}{*}{ Argila } & \multirow{2}{*}{ Silte } & \multicolumn{5}{|c|}{ Areia } & & \multirow{2}{*}{$\begin{array}{l}\text { Classe } \\
\text { textural }\end{array}$} \\
\hline & & & Fina & & Média & Grossa & Total & & \\
\hline $\mathrm{LVd}$ & 20,0 & 4,0 & 22,9 & & 35,7 & 17,4 & 76,0 & & Média \\
\hline
\end{tabular}

Departamento de Solos - FCA/UNESP - Botucatu/SP.

O experimento foi instalado em delineamento inteiramente casualizado com quatro repetições em esquema fatorial $(2 \times 6)+$ 1 , sendo o primeiro fator herbicidas aplicados em pré-emergência (tebuthiuron $1,0 \mathrm{~kg} \mathrm{ha}^{-1} \mathrm{e}$ atrazine $2,50 \mathrm{~kg} \mathrm{ha}^{-1}$ ) e o segundo intervalos de leitura do ETR, descrito posteriormente, além de uma testemunha sem herbicida. 
A aplicação dos herbicidas foi realizada por meio de um pulverizador estacionário instalado em laboratório e munido de barra contendo quatro pontas do tipo XR110.02. A pulverização foi realizada sobre pressão constante de 1,5 bar, pressurizado por ar comprimido, com consumo de calda de $200 \mathrm{~L}$ $\mathrm{ha}^{-1}$. A temperatura no momento da aplicação era de $25^{\circ} \mathrm{C}$ com umidade relativa de $70 \%$.

Após a aplicação dos tratamentos, as plantas foram transportadas para casa-devegetação onde ficaram até o término do ensaio, sendo realizadas as avaliações da taxa de transporte de elétrons e análise visual de fitointoxicação. A avaliação visual de intoxicação das plantas de cana-de-açúcar foi realizada 30 dias após a aplicação dos herbicidas através de uma escala de notas em porcentagem, onde " 0 " correspondia a nenhuma injúria e "100" a morte de plantas (SBCPD, 1995).

A leitura da taxa de transporte de elétrons (ETR) na porção mediana das folhas de cana-de-açúcar foi realizada com o fluorômetro portátil modelo Multi-Mode Chlorophyll Fluorometer OS5p (Opti-Sciences) nos intervalos de 16, 17, 19, 25, e 30 dias após a emergência.

A fluorescência no aparelho foi à fonte diodo com pico de luz vermelha no comprimento de onda de $660 \mathrm{~nm}$ sendo bloqueada radiações maiores que $690 \mathrm{~nm}$. A intensidade média da luz foi ajustada para o intervalo de 0 a $1 \mu \mathrm{Molm}^{-2} \mathrm{~s}^{-1}$, com o uso da lâmpada halogênica $35 \mathrm{~W}$. O feixe de luz foi opticamente monitorado no interior da câmara para corrigir as variações devido às mudanças na temperatura do ambiente no aparelho. Os sinais ópticos foram transferidos para a superfície da folha por uma trifurcação personalizada de fibra óptica, sendo de $2 \mathrm{~cm}^{2}$ a área iluminada utilizando protocolo Yield. A luz reemitida foi conduzida via fibra óptica para o aparelho através de três conectores que ligam na lateral do OS5p. As análises foram feitas seguindo a metodologia de Genty et al. (1989), avaliando a emissão da fluorescência da clorofila na face adaxial das folhas.

$\mathrm{O}$ protocolo Yield registra as medidas de quantum efetivo produzido no fotossistema II (PSII). Tanto a fonte de luz do sol quanto à luz artificial podem ser usadas para dirigir a fotossíntese.

Taxa de transporte de elétrons (ETR) $\mu$ Mols elétrons. $\mathrm{m}^{-2} \cdot \mathrm{s}^{-1}=(\mathrm{Y})$. $(\mathrm{PAR}) \cdot(0,84) \cdot(0,5)$ é equivalente a: (produção de quantum do PSII) $\mathrm{x}$ (medidas da radiação fotossinteticamente ativa medida em $\mu$ Mols elétrons. $\mathrm{m}^{-2} \cdot \mathrm{s}^{-1}$ ) x (coeficiente de absorção da folha) $\mathrm{x}$ (fração de luz absorvida pelo complexo antena do PSII). O ETR é uma medida da separação de cargas do centro de reação do PSII. Na equação são usados valores padrões, contudo, ambos os coeficientes de absorção e fração da luz absorvida pelo PSII podem ser trocados. Embora 0,84 seja um valor médio para muitas espécies de plantas, trabalhos têm mostrado que o coeficiente de absorção da folha pode variar com a qualidade da luz, espécie, conteúdo de clorofila e refletância da folha. Para a fração de luz que é absorvida pelo complexo antena do PSII os trabalhos mostram que ocorre variação de acordo com a espécie e estende de 0,42 a 0,60 (Laisk \& Loreto, 1996).

Como o parâmetro ETR, determina a taxa de transporte de elétrons no PSII, o uso do valor do ETR permite detectar o efeito da atuação do herbicida em nível de concentração de 0,5 micromoles $\mathrm{dm}^{-3}$, enquanto o método tradicional, que inclui a medição do parâmetro Fv/Fm, permite detectar apenas em um nível de concentração que é 100 vezes maior (Korres et al., 2003; Abbaspor et al., 2006), justificando o uso da metodologia empregada.

Os dados de eficiência fotossintética foram expressos em porcentagem da testemunha e foram submetidos à análise de 
variância. As médias comparadas pelo teste $t \mathrm{a}$ 10\% de significância (Velini et al., 2006).

\section{Resultados e discussão}

A síntese da análise de variância e teste de médias para os dados de ETR, analisados em função dos herbicidas e do tempo, estão expostos na Tabela 2.

Os efeitos isolados de tempo (dias) foram significativos a $1 \%$ de probabilidade e apenas aos 25 dias após a aplicação (DAA) dos herbicidas, foi apresentada a maior redução do ETR em relação aos demais intervalos avaliados. Já o efeito de cada herbicida, com tempo médio em dias, foi significativo também a $1 \%$, com o herbicida tebuthiuron apresentando maior redução do ETR. A interação dos dois fatores, tempo e herbicidas, também foi significativo a $1 \%$.

Tabela 2. Síntese da análise de variância e teste de médias para o ETR. Botucatu, SP, 2011.

\begin{tabular}{|c|c|}
\hline Tempo (DIAS APÓS APLICAÇÃ̃) & ETR \\
\hline 16 & $94,32 \mathrm{~b}$ \\
\hline 17 & $94,83 \mathrm{c}$ \\
\hline 19 & $92,39 \mathrm{~b}$ \\
\hline 25 & $88,75 \mathrm{a}$ \\
\hline 30 & $95,24 \mathrm{c}$ \\
\hline \multicolumn{2}{|l|}{ Herbicida (H) } \\
\hline Testemunha & $100,00 \mathrm{c}$ \\
\hline Atrazine & $96,89 \mathrm{~b}$ \\
\hline Tebuthiuron & $82,44 \mathrm{a}$ \\
\hline \multicolumn{2}{|l|}{ Teste $\mathbf{F}$} \\
\hline $\mathbf{T}$ & $10,70^{* *}$ \\
\hline $\mathbf{H}$ & $219,94 * *$ \\
\hline T $\times \mathbf{H}$ & $4,38 * *$ \\
\hline Coef. Variação (\%) & 4,29 \\
\hline
\end{tabular}


A partir da interação de todos os herbicidas com o período em dias (Tabela 3) observou-se, aos 16 DAA, que tebuthiuron apresentou com maior redução do ETR em comparação com atrazine. Com 17 DAA, o tebuthiuron manteve a inibição do transporte de elétrons em relação à atrazine. $\mathrm{O}$ mesmo ocorreu aos 19 e 25 DAA, entretanto, aos 30 DAA, ocorreu recuperação do ETR nas plantas que desenvolveram em solo submetido a aplicação de tebuthiuron.

Através do monitoramento do transporte de elétrons nas plantas de cana-deaçúcar verificou-se um efeito inicial maior de redução do ETR após aplicação do tebuthiuron. Isso se deve ao fato do ingrediente ativo do tebuthiuron atuar na inibição do transporte de elétrons no PSII, justamente onde é detectada, através das leituras do fluorômetro, a fluorescência emitida pelo aparato fotossintético após aplicação.

$\mathrm{O}$ atrazine também apresenta o mesmo mecanismo de ação, inibidor do PSII, entretanto, as plantas submetidas a tal produto não apresentaram essa intoxicação inicial como as plantas tratadas com tebuthiuron. Em trabalho desenvolvido por Girotto et al. (2010), com aplicação de atrazine em pósemergência também foi detectada baixa intoxicação, através do ETR, para a cultivar SP80 3280, quando comparado ao herbicida S-metolachlor.

Souza et al. (2009) buscaram avaliar a tolerância de cultivares de cana-de-açúcar a herbicidas aplicados em pós-emergência na soqueira da cultura. Foi detectada uma pequena redução no transporte de elétrons do PSII após aplicação de diuron + hexazinone, na fase inicial de desenvolvimento da cultura, mas não sendo suficiente para prejudicar a altura, estande, produção e qualidade tecnológica das cultivares estudadas.

Em trabalho desenvolvido por Dayan et al. (2009), foi monitorado o ETR em plantas de milho quando submetidas à aplicação de amicarbazone e atrazine. A taxa de transporte de elétrons manteve uma redução de aproximadamente $70 \%$ e $30 \%$ do ETR fotossintético com 24 horas após a aplicação, respectivamente, de amicarbazone e atrazine, sendo o milho mais tolerante a atrazine quando comparado ao amicarbazone, nas condições estudadas.

Dessa forma, o uso do fluorômetro torna-se uma ferramenta interessante de identificação precoce das injurias fisiológica (de 25 a 30 dias após aplicação para os herbicidas em estudo no trabalho), fato esse não identificado através de análise visual de fitointoxicação.

Algumas oscilações nos valores de ETR para o tratamento tebuthiuron devem possivelmente a algum efeito do ambiente. As pequenas oscilações do ETR ocorridas ao longo do período avaliado, devem-se às condições do ambiente, visto que a capacidade fotossintética das plantas pode ser alterada por estresses bióticos ou abióticos, como mudanças na temperatura, radiação, deficiência hídrica, etc (Bown et al., 2002). 
Tabela 3. Respostas da taxa de transporte de elétrons (ETR), em função da interação do tempo após aplicação e herbicidas utilizados em pré-emergência em cana-de-açúcar. Botucatu, SP, 2011.

\begin{tabular}{cccccc}
\hline \multirow{2}{*}{ Herbicidas } & \multicolumn{5}{c}{ Período (Dias Após Aplicação) } \\
\cline { 2 - 5 } & $\mathbf{1 6}$ & $\mathbf{1 7}$ & $\mathbf{1 9}$ & $\mathbf{2 5}$ & $\mathbf{3 0}$ \\
\hline Testemunha & $100,00 \mathrm{Ab}$ & $100,00 \mathrm{Ab}$ & $100,00 \mathrm{Ac}$ & $100,00 \mathrm{Ac}$ & $100,00 \mathrm{Ab}$ \\
Atrazine & $98,23 \mathrm{Bb}$ & $97,56 \mathrm{Bb}$ & $95,78 \mathrm{ABb}$ & $93,42 \mathrm{Ab}$ & $99,45 \mathrm{Ba}$ \\
Tebuthiuron & $84,73 \mathrm{BCa}$ & $86,92 \mathrm{Ca}$ & $81,40 \mathrm{Ba}$ & $72,85 \mathrm{Aa}$ & $86,30 \mathrm{Ca}$ \\
\hline
\end{tabular}

**Médias seguidas por letras maiúsculas distintas diferem estatisticamente entre si pelo teste $t(p>0,10)$, na linha.

*Médias seguidas por letras minúsculas distintas diferem estatisticamente entre si pelo teste $t(p>0,10)$, na coluna.

Em relação à redução do transporte de elétrons, a aplicação do tebuthiuron apresentou maior redução quando comparado ao herbicida atrazine. Entretanto, esta redução não afetou o desenvolvimento inicial da canade-açúcar, o que foi verificado pela análise de fitointoxicação.

Os dados médios para cada tratamento com herbicida e os sintomas de fitointoxicação (Figura 1) foram observados nas plantas tratadas com atrazine com aproximadamente $10 \%$ de fitointoxicação, e $8 \%$ para o tebuthiuron, não diferindo significativamente entre si.

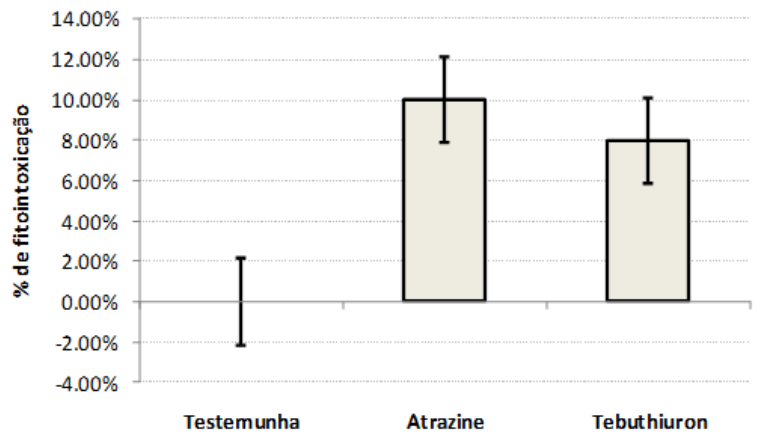

Figura 1. Porcentagem de fitointoxicação aos 30 DAA dos produtos. Botucatu, SP, 2011.

As plantas de cana-de-açúcar apresentaram diferente sensibilidade aos tratamentos, com destaque para o tebuthiuron, que apresentou maior redução do ETR nas condições e períodos estudados.

De modo geral, a metodologia empregada com o uso do fluorômetro mostrou-se adequada para verificar a ação de herbicidas inibidores do fotossistema II, através da verificação instantânea do transporte de elétrons, fase essa inicial da fotossíntese nas plantas. E, com a aplicação do tebuthiuron na cultivar de cana-de-açúcar SP80 3280 foi verificada a intoxicação na cultura mesmo antes de qualquer detecção visual da atuação do herbicida nas plantas.

\section{Conclusões}

A cultivar de cana-de-açúcar SP803280 apresentou uma boa seletividade aos herbicidas tebuthiuron e atrazine aplicados em pré-emergência. As maiores reduções do ETR foram observadas nas plantas que se desenvolveram em solo que recebeu a aplicação de tebuthiuron, sendo uso do fluorômetro uma alternativa para detectar precocemente as injúrias de herbicidas na cultura da cana-de-açúcar. 


\section{Referências}

ABBASPOOR, M.; TEICHER H.B.; STREIBIG J.C. The effect of root-absorbed PSII inhibitors on Kautsky curve parameters in sugar beet. Weed Research, v.46, n.3, p.226-235, 2006.

BOWN, A.W.; HALL, D.E.; MACGREGOR, K.B. Insect footsteps on leaves stimulate the accumulation of 4-aminobutyrate and can be visualized through increased chlorophyll fluorescence and superoxide production. Plant Physiology, v.129, n.4, p.1430-1434, 2002.

BREITENBACH, J.; ZHU, C.; SANDMAN, G. Bleaching herbicide norflurazon inhibits phytoene desaturase by competition with the cofactors. Journal of Agricultural and Food Chemmistry, v.49, n.11, p.5270-5272, 2001.

DAYAN, F.E.; TRINDADE, M.L.B.; VELINI, E.D. Amicarbazone, a new photosystem II inhibitor. Weed Science, v.57, p.579-583, 2009.

FREITAS, S.P. et al. Controle químico de Rottboelia exaltata em cana-de-açúcar. Planta Daninha, v.22, n.3, p. 461-466, 2004.

GENTY, B.; BRIANTAIS, J.M.; BAKER, N.R. The relationship between the quantum yield of photosynthetic electron-transport and quenching of chlorophyll fluorescence. Biochimica et Biophysica Acta, v.990, n.1, p.87-92, 1989.

GIROTTO, M. et al. Eficiência fotossintética da cana-de-açúcar após aplicação dos herbicidas S-metalachor e atrazine em pósemergência. Revista Brasileira de Herbicidas, v.9, n.3, p.109-116, 2010.

IRELAND， C.R.; PERCIVAL， M.P.; BAKER, N.R. Modification of the induction of photosynthesis in wheat by glyphosate, an inhibitor of amino acid metabolism. Journal Experimental Botany, v.37, n.176, p.299308, 1986.
KORRES, N.E., FROUD-WILLIAMS, R.J, MOSS, S.R. Chlorophyll fluorescence technique as a rapid diagnostic test of the effects of the photosynthetic inhibitor chlortoluron on two winter wheat cultivars. Annals Applied Biology, v.143, n.7, p.53-56, 2003.

KRAUSE, G.H.; WINTER, K. Photoinhibition of photosynthesis in plants growing in natural tropical forest gaps: a chlorophyll fluorescence study. Botanica Acta, v.109, n.6, p.456-462, 1996.

LAISK, A.; LORETO, F. Determining photosynthetic parameters from leaf $\mathrm{CO}_{2}$ exchange and chlorophyll fluorescence. Ribulose-1,5-bisphosphate carboxylase/ oxygenase specificity factor, dark respiration in the light, excitation distribution between photosystems, alternative electron transport rate, and mesophyll diffusion resistance. Plant Physiology, v.110, n.3, p.903-912, 1996.

MAXWELL, K.; JOHNSON, G.N. Chlorophyll fluorescence: a practical guide. Journal of Experimental Botanica, v.51, n.345, p.659-668, 2000.

NEGRISOLI, E. et al. Seletividade de herbicidas aplicados em pré-emergência na cultura da cana-de-açúcar tratada com nematicidas. Planta Daninha, v.22, n.4, p.567-575, 2004.

RODRIGUES, B.N.; ALMEIDA, F.S. Guia de Herbicidas, 5.ed. Londrina, PR, 2005.

SOCIEDADE BRASILEIRA DA CIÊNCIA DAS PLANTAS DANINHAS. Procedimentos para instalação, avaliação e análise de experimentos com herbicidas. Londrina: SBCPD, 1995. $42 \mathrm{p}$. 
SOUZA, J.R. et al. Tolerância de cultivares de cana-de-açúcar a herbicidas aplicados em pós-emergência. Bragantia, v.68, n.14, p.873-884, 2009.

VELINI, E.D. et al. Interferência de plantas daninhas na cultura do milho. I - efeito do número de repetições sobre a precisão dos resultados obtidos. Planta Daninha, v.24, n.3, p.435-442, 2006.

YOUNG, A.L.; FRANK, H.A. Energy transfer reactions involving carotenoids: quenching of chlorophyll fluorescence. Journal of Photochemistry and Photobiology B: Biology. v.36, n.1, p.3-15, 1996. 